\title{
Mitteilungen
}

\section{Ernst Lewy}

$1881-1966$

Einen Nachruf auf E. LEwy zu schreiben ist u.a. deshalb schwierig, weil es dabei nicht nur darum gehen kann, auf seine Verdienste hinzuweisen, sondern gleichzeitig darum, einer Vernachlässigung seiner Arbeiten in der wissenschaftlichen Praxis, über die der Verstorbene mir gegenüber, aber auch in seinen Veröffentlichungen immer wieder klagte ${ }^{\boldsymbol{x}}$, entgegenzuwirken.

Der Grund, dass Lewy trotz des hohen Niveaus seiner Arbeiten eigentlich zeit seines Lebens verhältnismässig unbeachtet und auch, schlimmer, unverstanden geblieben ist, ist, so will mir scheinen, hauptsächlich der, dass er sich niemals der gängigen Sprachwissenschaft eingefügt hat, immer ein selbständiger Denker war. In erster Linie Schüler F. N. Fiscks (aber auch W. Schulzes, Brugmaxxs, Hirts, Delbrücks und anderer Grössen der letzten Blütezeit deutscher Sprachwissenschaft, in der Finnougristik $\mathrm{H}$. WinkLens), teilte er somit das Schicksal seines Lehrers und der übrigen Glieder einer schmalen Kette deutscher allgemeiner Sprachwissenschaftler, an deren Beginn W. v. Humbolut steht (der ja anch, wiewohl hochgeschätzt, eigentlich kaum eine Rolle in der Praxis spielte, bis ihm Cuomsky wieder Geltung verschuf).

Getreu diesem Lehrer ${ }^{2}$ trieb Lewy vorzüglich das, was man heute "synchrone Sprachwissenschaft" nennt, nur in einem anderen als dem üblichen Sinn, weswegen er bci den späteren "Strukturalisten" auch nicht bekannt wurde. Seine Arbeitsmethode bestand darin, eine Sprache in ihrer Systematik so zu erfassen, dass sich bestimmte, typologisch ausschlaggebende Züge in ihr herauskristallisierten, und Beziehungen zwischen diesen Zügen herzustellen, "veranschaulichen", wie er es nannte, oder "charakterisieren". So entstand eine Reihe von Sprach-

1 Vgl. z.B. "Zur Betonung ...., S. 7.

${ }^{2}$ Aufschlussreich ist L.s. verständnisvoll-kritische Bemerkung, Kleine Schriften (KS) 93, dass FINCK im Eifer des Kampfes ... die historische Sprachwissenschaft aus der Wissenschaft ausschloss." Finck starb 1910. 
skizzen, knapp, präzis, fast aphoristisch, klar, freilich ohne Vorkenntnisse schwer verstehbar, des Ungarischen, Russischen, Mordwinischen, Awarischen, Quiché und eine ganze Serie in seinem Buch "Der Bau der europäischen Sprachen» (seinem "Lebenswerk").

Solche Sprachcharakteristiken, in denen übrigens eine Menge Arbeit steckt, gab es ja schon vor ihm, wenn auch, wie mir scheint, nicht in dieser Meisterschaft, aber was L. daraus gemacht hat, ist völlig originell. Zunächst löste er sich sehr schnell von den psychologischen Erwägungen FIxcks, die ja kennzeichnend für diese Zeit waren, d.h. in diesem Fall der Inbeziehungsetzung der Sprachtypen mit der Lehre von den Temperamenten (; gegen die Psychologismen vgl. KS 332: "Mit dürren Worten sage ich, dass die Erklärungen syntaktischer Erscheinungen meist keine sind." Dass L. sich immer wieder an die Frage der Beziehungen bestimmter Spracherscheinungen mit bestimmten Arten zu denken herantastete, ist ja eine andere Sache; vgl. KS 171, Fn. 2). Dann versuchte er eine Verknüpfung dieser Art von Typologie mit der heute sogenannten "arealen Linguistik", die damals allerdings weitgehend unbekannt war (von anderen Gesichtspunkten aus wurde dieser nun etablierte Wissenschaftszweig von Scнuchardt, der Prager Schule und Boas begründet, vgl. Jakorsos, IJAL 10). D.h. bestimmte, im Laufe der "Charakterisierung» abstrahierte Züge einer Sprache werden über deren Territorium hinaus verfolgt, wiedergefunden (oder nicht), woraus sich eine nelle (geographische) Einteilung der Sprachen ergibt, die mit der genealogischen nicht identisch ist (besser: nicht zu sein bralucht), was am besten im "Bau ..." zu sehen ist.

Darüber hinaus hat aber I. schon früh die Tragweite seiner Betrachtungsweise auch für die Sprachgeschichte erkannt, vgl. z.B., zum Ungarischen, KS 491 (1924). Und zwar vollzog er diese geschichtliche Auswertung auf zwei, letztlich eng zusammengehörige Weisen: Die erste ist der Versuch, die Geschichtlichkeit einer Sprache zu erfassen, d.h. bei ihm das Aufdecken einer Komposition von typologischen Zügen, die die Sprache im Laufe ihrer (reschichte sich angeeirnet hat, durch das Zusammentreffen ihrer Sprecher mit Sprechern anderer Sprachen, durch "Sprachmischung" (vgl. dazu die schon 1913 geschriebene Abhandlung, KS 1 ff.), die ja einerseits durch Nachbarschaft, andererseits durch sog. Substratwirkung (ein Wort, das L. nicht leiden konnte) vor sich gehen kann, wie etwa im Falle des Finnisch-Ugrischen und Russischen (s. vor allem KS 341 ff.). Die andere Weise, das "deskriptiv» Erkannte für die Sprachgeschichte zu verwerten, besteht bei L. in der Interpretation der Verbreitung typologischer Züge auf der Sprachen- 
karte. Dabei erkannte er einerseits die geographische Exzessivität der relativ reinen Sprachtypen (Fiscks "Haupttypen des Sprachbaus») auf Kosten der durch diese an den Rand der Karte gedrängten Sprachen mit stark gemischtem Typus; andererseits verband er typologische Eigenheiten ebendieser Sprachen miteinander, was historische Perspektiven von ungeahnter Wichtigkeit öffnet, mit Ergebnissen, die sich etwa für den eurasiatischen Raum, unabhängig, mehr und mehr durchsetzen (vgl. KS $7 \vec{f}$ ff., 612 ff., 9 ff. u.a.).

Obgleich L. der eigentliche Begründer der Fimnougristik in Deutschland ist (Schlachter, Steinitz, Bouda, Bussenius, J Acobsons u.a. waren seine Schüler), war sie für ihn nur Hauptarbeitsfeld, im Prinzip war er immer allgemeiner Sprachwissenschaftler. Dass dies ein richtiger und fortschrittlicher Standpunkt war, wird heute, da alle einzelnen "Philologrien" mehr und mehr gezwungen sind, sich als Spezialgebiete innerhalb der allgemeinen sprachwissenschaft zu verstehen, kalum jemand bestreiten. L.s Versuch einer Integration der Finnougristik in die allgemeine Sprachwissenschaft ist damit heute auch das methodologisch Wichtigste und Interessinteste an seinen Arbeiten für den Finnougristen. Das bedeutet aber auch, dass er nicht nur die Spezialarbeiten zu lesen hat: I.S Arbeiten bilden in sich eine Einheit, Gedanken aus früheren Abhandlungen werden immer wieder aufgegriffen, weitergesponnen, in neue Zusammenhänge gestellt. Die Untersuchungen der Akzentverhältnisse, um nur ein Beispiel zu nennen, im Mordwinischen, Ostjakischen, Labourdinisch-Baskischen, Awarischen, Quiché gehören zusammen. Dass in Diskussionen um die fiugr. Urheimat kaum jemals auch nur erwähnt wird, dass L. den einzigen Versuch einer Bestimmung mit rein linguistischen Jitteln unternommen hat, ist vielleicht daranf zurückzuführen, dass er in einer Abhandlung über die idg. Urheimat erschien (KS $200 \mathrm{ff}$.).

An Spezialarbeiten verdankt die Finnougristik LEwy die schwer lesbare ${ }^{1}$ "Tscheremissische Grammatik" ${ }^{2}$, im Wesentlichen die Beschreibung eines wiesentscheremissischen Idiolekts, nebst Texten ("Tscheremissische Texte" 1925-6), die bestechend unanständigen und (deshalb?) kaum je berïck-

${ }^{1}$ L. führte dies darauf zurück, dass es ihm nicht möglich war, das dazugehörige Wörterbuch zu veröffentlichen. Das Manuskript befindet sich $\mathrm{m}$. W. in den Händen W. VEENKERS.

${ }^{2}$ Gerade weil heute selbstverständlich, folgende Notiz L.s: "... die Erkenntnis, dass die Grammatik eine autonome Wissenschaft ist, und eine Grammatik ein Buch, das irgendwelche 7wecke ausser sich selbst ebensowenig kennt, als ein Lehrbuch der Mathematik, ist noch wenig verbreitet, am wenigsten wohl unter den Verfassern von Grammatiken" (KS 331,1925$)$. 
sichtigten "Mordwinischen Märchen in erzjanischem Dialekte" (1931), die noch in hohem Alter verfasste, minutiöse Abhandlung "Zur Betonung des Erdzja-Mordwinischen von Orkino" (1961) (eine Frage übrigens, die auch die Prager Schule beschäftigte). Besonders aber möchte ich seine Habilitationsschrift "Zur finnisch-ugrischen Wort- und Satzverbindung" (1911) hervorheben.

Nicht zuletzt gibt es von L. eine ganze Menge geistreicher, jenseits jeder Routine liegender Etymologien, die sich freilich, da L. an Etymologien hauptsächlich die semantische Seite interessierte, in Kompendien nicht so leicht verwerten lassen und sich wohl deshalb geringer Beachtung erfreuen.

Ich bin mir bewusst, dass ich mit diesen knappen Worten kaum Andeutungen über die Bedeutung der Arbeit ${ }^{1}$ des Verstorbenen machen konnte ${ }^{2}$ und kann deshalb nur hoffen, dass dadurch der eine oder andere Wissenschaftler angeregt wird, diesen grossen, unkonventionellen Mann, den, die private $\mathrm{Be}^{-}$ merkung sei erlaubt, Güte und Verzicht auf unverbindliche Umgangsformen, stete Bereitschaft zum Umdenken wie Kompromisslosigkeit in der Sache gleichermassen auszeichneten, dessen Schriften immer (sogar da, wo sachliche Fehler ${ }^{3}$ vorliegen) anregend und wichtig sind, eindringlicher zu lesen.

HaRTMut Ka'TZ

\section{E. A. Virtanen}

\section{$1897-1970$}

Dr. Erik Anton Virtanen war von 1953--1964 ausserordentlicher Professor für finnisch-ugrische Ethnologie an der Universität Helsinki. Er vertrat in seinen Forschungen und an der Universität soziale Ethnologie und definierte selber in seiner Dissertation 1950 sein Gebiet als Ethnosoziologie. In seinen umfassenden wissenschaftlichen Werken (Veröffentlichungen vom Jahre 1918 an) untersuchte er Struktur und Funktion

\footnotetext{
${ }^{1}$ L. war auch auf literarischem Gebiet eine Kapazität. Wenige wissen, dass die erste Lenz-Gesamtausgabe von ihm stammt.

2 Ein besserer Kenner der Arbeiten L.s ist Loнmans, weswegen ich auf UAJb XXVIII, 1 ff. und sein Buch "Philosophie und Sprachwissenschaft" (passim) verweisen möchte.

${ }^{3}$ Es ist immer zu bedenken, dass L. in seinem dreissigjährigen Exil in Dublin, nach dem Verlust des grössten Teils seiner Bibliothek, unter sehr erschwerten Bedingungen arbeiten musste.
} 